\title{
A Study of Caputo-Hadamard-Type Fractional Differential Equations with Nonlocal Boundary Conditions
}

\author{
Wafa Shammakh \\ Faculty of Science, King Abdulaziz University, AL Faisaliah Campus, Jeddah, Saudi Arabia \\ Correspondence should be addressed to Wafa Shammakh; wshammakh@kau.edu.sa \\ Received 17 February 2016; Accepted 16 March 2016 \\ Academic Editor: Jozef Banas
}

Copyright ( 2016 Wafa Shammakh. This is an open access article distributed under the Creative Commons Attribution License, which permits unrestricted use, distribution, and reproduction in any medium, provided the original work is properly cited.

Existence and uniqueness results of positive solutions to nonlinear boundary value problems for Caputo-Hadamard fractional differential equations by using some fixed point theorems are presented. The related Green's function for the boundary value problem is given, and some useful properties of Green's function are obtained. Example is presented to illustrate the main results.

\section{Introduction}

In this paper, we study the existence and uniqueness of positive solutions for the $m$-point boundary value problems for Caputo-Hadamard fractional differential equations of the form

$$
\begin{aligned}
& { }_{H}^{C} D^{\alpha} x(t)+f(t, x(t))=0, \\
& t \in[1, e], n-1<\alpha \leq n, n>2, \\
& { }_{H}^{C} D x(1)=\sum_{i=1}^{m-2} \beta_{i}{ }_{H}^{C} D x\left(\eta_{i}\right), \\
& { }_{H}^{C} D^{2} x(1)={ }_{H}^{C} D^{3} x(1)=\cdots={ }_{H}^{C} D^{(n-1)} x(1)=0, \\
& x(e)=\sum_{i=1}^{m-2} \gamma_{i} x\left(\eta_{i}\right),
\end{aligned}
$$

where ${ }_{H}^{C} D^{\alpha}$ is the Caputo-Hadamard fractional derivative of order $n-1<\alpha \leq n, \sum_{i=1}^{m-2} \gamma_{i}<1, \sum_{i=1}^{m-2} \beta_{i}<1, \eta_{i} \in(1, e)$ for all $i=1,2, \ldots, m-2, \eta_{1}<\eta_{2}<\cdots<\eta_{m-2}$ and $f$ : $[1, e] \times[0, \infty) \rightarrow[0, \infty)$.

The derivative is a kind of fractional derivatives attributed to Hadamard in 1892 [1]; this fractional derivative differs from the Riemann-Liouville and Caputo fractional derivatives in the sense that the kernel of the integral contains a logarithmic function of arbitrary exponent. The Riemann-Liouville and
Hadamard derivative have their own disadvantages as well, one of which is the fact that the derivative of a constant is not equal to zero in general. The subject of Hadamard-type fractional differential equations has received much attention by many researchers. Some new results on the existence of solutions for a fractional boundary value problem involving Hadamard-type fractional differential inclusions and integral boundary conditions can be found in [2].

In [3], Tariboon et al. studied the existence and uniqueness of solutions to the boundary value problems consisting of a fractional differential equation of Riemann-Liouville type, subject to the Hadamard fractional integral equations. Thiramanus et al. [4] investigated the existence and uniqueness of solutions for a Hadamard fractional differential equations with nonlocal fractional integral boundary conditions. Ahmad and Ntouyas [5, 6] studied the existence results for a boundary value problem of nonlinear fractional hybrid differential inclusions of Hadamard type with Dirichlet boundary condition and for a coupled system of Hadamard fractional differential equations and Hadamard-type integral boundary conditions, respectively.

Jarad et al. [7] modified the Hadamard fractional derivative into a more suitable one having physically interpretable initial conditions similar to the ones in the Caputo setting.

Basic definitions and properties of fractional calculus and Hadamard-type fractional calculus can be found in [8-10].

The idea of this paper is to demonstrate sufficient conditions on existence and uniqueness of positive solutions 
to nonlinear boundary value problems (1) for modified Hadamard fractional (Caputo-Hadamard) differential equations, by using Banach's fixed point theorem, Leray-Schauder nonlinear alternative theorem for single valued maps, Krasnoselskii's fixed point theorem, and some properties of the Green function.

\section{Preliminaries}

In this section, we introduce some notations and definitions of Hadamard-type fractional calculus.

Definition 1 (see $[11,12])$. The Hadamard derivative of fractional order $\alpha$ for a function $g:[1, \infty) \rightarrow \mathbb{R}$ is defined as

$$
\begin{array}{r}
D^{\alpha} g(t)=\frac{1}{\Gamma(n-\alpha)}\left(t \frac{d}{d t}\right)^{n} \int_{1}^{t}\left(\log \frac{t}{s}\right)^{n-\alpha-1} \frac{g(s)}{s} d s, \\
n-1<\alpha<n, n=[\alpha]+1,
\end{array}
$$

where $[\alpha]$ denotes the integer part of the real number $\alpha$ and $\log (\cdot)=\log _{e}(\cdot)$.

Definition 2 (see $[11,12])$. The Hadamard fractional integral of order $\alpha \in \mathbb{R}^{+}$of a function $g(x), \forall x>0$, is defined as

$$
\begin{aligned}
& I_{a+}^{\alpha} g(x)=\frac{1}{\Gamma(\alpha)} \int_{a}^{x}\left(\log \frac{x}{t}\right)^{\alpha-1} \frac{g(t)}{t} d t, \\
& I_{b-}^{\alpha} g(x)=\frac{1}{\Gamma(\alpha)} \int_{x}^{b}\left(\log \frac{t}{x}\right)^{\alpha-1} \frac{g(t)}{t} d t .
\end{aligned}
$$

Definition 3 (see $[1,7]$ ). Let $\Re(\alpha) \geq 0, n=[\Re(\alpha)+1]$, and $g \in A C_{\delta}^{n}[a, b], 0<a<b<\infty$. Then

$$
\begin{aligned}
& { }^{C} D_{a+}^{\alpha} g(x)=D_{a+}^{\alpha}\left[g(x)-\sum_{k=0}^{n-1} \frac{\delta^{k} g(a)}{k !}\left(\log \frac{t}{a}\right)^{k}\right](x), \\
& { }^{C} D_{b-}^{\alpha} g(x) \\
& =D_{b-}^{\alpha}\left[g(x)-\sum_{k=0}^{n-1} \frac{(-1)^{k} \delta^{k} g(b)}{k !}\left(\log \frac{b}{t}\right)^{k}\right](x) .
\end{aligned}
$$

Here $\mathfrak{R}(\alpha) \geq 0, n=[\Re(\alpha)+1], 0<a<b<\infty$ and

$$
\begin{aligned}
g & \in A C_{\delta}^{n}[a, b]=\left\{g:[a, b] \longrightarrow \mathbb{C}: \delta^{(n-1)} g(x)\right. \\
& \left.\in A C[a, b], \delta=x \frac{d}{d x}\right\} .
\end{aligned}
$$

In particular, if $0<\mathfrak{R}(\alpha)<1$, then

$$
\begin{aligned}
& { }^{C} D_{a+}^{\alpha} g(x)=D_{a+}^{\alpha}[g(x)-g(a)](x), \\
& { }^{C} D_{b-}^{\alpha} g(x)=D_{b-}^{\alpha}[g(x)-g(b)](x) .
\end{aligned}
$$

Theorem 4 (see $[1,7]$ ). Let $\mathfrak{R}(\alpha) \geq 0, n=[\Re(\alpha)+1]$ and $g \in$ $A C_{\delta}^{n}[a, b], 0<a<b<\infty$. Then ${ }^{C} D_{a+}^{\alpha} g(x)$ and ${ }^{C} D_{b-}^{\alpha} g(x)$ exist everywhere on $[a, b]$ and

(a) if $\alpha \notin \mathbb{N}_{0}$,

$$
\begin{aligned}
{ }^{C} D_{a+}^{\alpha} g(x) & =\frac{1}{\Gamma(n-\alpha)} \int_{a}^{x}\left(\log \frac{x}{t}\right)^{n-\alpha-1} \delta^{n} g(t) \frac{d t}{t} \\
& =I_{a+}^{n-\alpha} \delta^{n} g(x), \\
{ }^{C} D_{b-}^{\alpha} g(x) & =\frac{(-1)^{n}}{\Gamma(n-\alpha)} \int_{x}^{b}\left(\log \frac{t}{x}\right)^{n-\alpha-1} \delta^{n} g(t) \frac{d t}{t} \\
& =(-1)^{n} I_{a+}^{n-\alpha} \delta^{n} g(x),
\end{aligned}
$$

(b) if $\alpha=n \in \mathbb{N}_{0}$,

$$
\begin{aligned}
& { }^{C} D_{a+}^{\alpha} g(x)=\delta^{n} g(x), \\
& { }^{C} D_{b-}^{\alpha} g(x)=(-1)^{n} \delta^{n} g(x) .
\end{aligned}
$$

In particular,

$$
{ }^{C} D_{a+}^{0} g(x)={ }^{C} D_{b-}^{0} g(x)=g(x) .
$$

Lemma 5 (see $[1,7]$ ). Let $\mathfrak{R}(\alpha) \geq 0, n=[\mathfrak{R}(\alpha)+1]$, and $g \in C[a, b]$.

If $\mathfrak{R}(\alpha) \neq 0$ or $\alpha \in \mathbb{N}$, then

$$
\begin{aligned}
& { }^{C} D_{a+}^{\alpha}\left(I_{a+}^{\alpha} g\right)(x)=g(x), \\
& { }^{C} D_{b-}^{\alpha}\left(I_{b-}^{\alpha} g\right)(x)=g(x) .
\end{aligned}
$$

Lemma 6 (see $[1,7]$ ). Let $g \in A C_{\delta}^{n}[a, b]$ or $C_{\delta}^{n}[a, b]$ and $\alpha \in$ $C$, and then

$$
\begin{aligned}
I_{a+}^{\alpha}\left({ }^{C} D_{a+}^{\alpha} g\right)(x)= & g(x)-\sum_{k=0}^{n-1} \frac{\delta^{k} g(a)}{k !}\left(\log \frac{t}{a}\right)^{k}, \\
I_{b-}^{\alpha}\left({ }^{C} D_{b-}^{\alpha} g\right)(x)= & g(x) \\
& -\sum_{k=0}^{n-1} \frac{(-1)^{k} \delta^{k} g(a)}{k !}\left(\log \frac{b}{t}\right)^{k} .
\end{aligned}
$$

Lemma 7 (see $[1,7]$ ). For $n-1<\alpha \leq n, n>2$, and $h \in$ $C([1, e], \mathbb{R})$, the unique solution of the problem

$$
\begin{aligned}
{ }_{H}^{C} D^{\alpha} x(t)+h(t) & =0, \quad t \in[1, e], \\
{ }_{H}^{C} D x(1) & =\sum_{i=1}^{m-2} \beta_{i}{ }_{H}^{C} D x\left(\eta_{i}\right), \\
{ }_{H}^{C} D^{2} x(1) & ={ }_{H}^{C} D^{3} x(1)=\cdots={ }_{H}^{C} D^{(n-1)} x(1) \\
& =0, \\
x(e) & =\sum_{i=1}^{m-2} \gamma_{i} x\left(\eta_{i}\right)
\end{aligned}
$$

is given by

$x(t)$

$$
=\int_{1}^{e}\left[G(t, s)+H\left(t, s ; \eta_{1}, \eta_{2}, \ldots, \eta_{m-2}\right)\right] h(s) \frac{d s}{s},
$$


where

$$
\begin{aligned}
& G(t, s)=\frac{1}{\Gamma(\alpha)} \begin{cases}\left(\log \frac{e}{s}\right)^{\alpha-1}-\left(\log \frac{t}{s}\right)^{\alpha-1}, & 1 \leq s \leq t \leq e, \\
\left(\log \frac{e}{s}\right)^{\alpha-1}, & 1 \leq t \leq s \leq e,\end{cases} \\
& H\left(t, s ; \eta_{1}, \eta_{2}, \ldots, \eta_{m-1}\right) \\
& = \begin{cases}\frac{\sum_{i=1}^{m-2} \gamma_{i}\left[(\log (e / s))^{\alpha-1}-\left(\log \left(\eta_{i} / s\right)\right)^{\alpha-1}\right]}{\delta_{2} \Gamma(\alpha)}+\frac{\left(\mu-\delta_{2}(\log t)\right) \sum_{i=1}^{m-2} \beta_{i}\left(\log \left(\eta_{i} / s\right)\right)^{\alpha-2}}{\delta_{1} \delta_{2} \Gamma(\alpha-1)}, & 1 \leq s \leq \eta_{i}, i=1,2, \ldots, m-1, \\
\frac{\sum_{i=1}^{m-2} \gamma_{i}(\log (e / s))^{\alpha-1}}{\delta_{2} \Gamma(\alpha)}, & \eta_{i} \leq s \leq e, i=1,2, \ldots, m-1,\end{cases} \\
& \delta_{1}=1-\sum_{i=1}^{m-2} \beta_{i}, \delta_{2}=1-\sum_{i=1}^{m-2} \gamma_{i}, \mu=1-\sum_{i=1}^{m-2} \gamma_{i}\left(\log \eta_{i}\right) .
\end{aligned}
$$

Proof. In view of Lemma 6, the solution of the Hadamard differential equation (12) can be written as

$$
\begin{aligned}
& x(t)=-{ }_{H} I^{\alpha} h(t)+c_{0}+c_{1} \log t+c_{2}(\log t)^{2} \\
& +c_{3}(\log t)^{3}+\cdots+c_{n-1}(\log t)^{n-1}, \\
& { }_{H}^{C} D x(t)=-{ }_{H} I^{\alpha-1} h(t)+c_{1}+2 c_{2} \log t+3 c_{3}(\log t)^{2} \\
& \quad+\cdots+(n-1) c_{n-1}(\log t)^{n-2}, \\
& { }_{H}^{C} D^{2} x(t)=-{ }_{H} I^{\alpha-2} h(t)+2 c_{2}+6 c_{3} \log t+\cdots \\
& +(n-1)(n-2) c_{n-1}(\log t)^{n-3}, \\
& \quad \vdots \\
& { }_{H}^{C} D^{(n-1)} x(t)=-{ }_{H} I^{\alpha-n+1} h(t)+(n-1) ! c_{n-1} .
\end{aligned}
$$

$$
\begin{aligned}
c_{2}= & c_{3}=\cdots=c_{n-1}=0, \\
c_{1}= & -\frac{1}{\delta_{1}} \sum_{i=1}^{m-2} \beta_{i} I^{\alpha-1} h\left(\eta_{i}\right), \\
c_{0}= & \frac{1}{\delta_{2}}\left[I^{\alpha} h(e)-\sum_{i=1}^{m-2} \gamma_{i} I^{\alpha} h\left(\eta_{i}\right)\right] \\
& +\frac{\mu}{\delta_{1} \delta_{2}} \sum_{i=1}^{m-2} \beta_{i} I^{\alpha-1} h\left(\eta_{i}\right) .
\end{aligned}
$$

Substituting the values of $c_{i}, i=0,1, \ldots, n-1$, we obtain

$$
\begin{gathered}
x(t)=-I^{\alpha} h(t)+\frac{1}{\delta_{2}}\left[I^{\alpha} h(e)-\sum_{i=1}^{m-2} \gamma_{i} I^{\alpha} h\left(\eta_{i}\right)\right] \\
+\frac{\left(\mu-\delta_{2}(\log t)\right)}{\delta_{1} \delta_{2}}\left(\sum_{i=1}^{m-2} \beta_{i} I^{\alpha-1} h\left(\eta_{i}\right)\right)=-I^{\alpha} h(t)
\end{gathered}
$$

$$
\begin{aligned}
& +I^{\alpha} h(e)+\frac{1}{\delta_{2}}\left[\left(1-\delta_{2}\right) I^{\alpha} h(e)-\sum_{i=1}^{m-2} \gamma_{i} I^{\alpha} h\left(\eta_{i}\right)\right] \\
& +\frac{\left(\mu-\delta_{2}(\log t)\right)}{\delta_{1} \delta_{2}}\left(\sum_{i=1}^{m-2} \beta_{i} I^{\alpha-1} h\left(\eta_{i}\right)\right)=-I^{\alpha} h(t) \\
& +I^{\alpha} h(e)+\frac{1}{\delta_{2}}\left[\sum_{i=1}^{m-2} \gamma_{i} I^{\alpha} h(e)-\sum_{i=1}^{m-2} \gamma_{i} I^{\alpha} h\left(\eta_{i}\right)\right] \\
& +\frac{\left(\mu-\delta_{2}(\log t)\right)}{\delta_{1} \delta_{2}}\left(\sum_{i=1}^{m-2} \beta_{i} I^{\alpha-1} h\left(\eta_{i}\right)\right)=-\frac{1}{\Gamma(\alpha)} \\
& \cdot \int_{1}^{t}\left(\log \frac{t}{s}\right)^{\alpha-1} h(s) \frac{d s}{s}+\frac{1}{\Gamma(\alpha)} \\
& \cdot \int_{1}^{e}\left(\log \frac{e}{s}\right)^{\alpha-1} h(s) \frac{d s}{s}+\frac{1}{\delta_{2} \Gamma(\alpha)} \sum_{i=1}^{m-2} \gamma_{i} \\
& \cdot\left[\int_{1}^{e}\left(\log \frac{e}{s}\right)^{\alpha-1} h(s) \frac{d s}{s}\right. \\
& \left.-\int_{1}^{\eta_{i}}\left(\log \frac{\eta_{i}}{s}\right)^{\alpha-1} h(s) \frac{d s}{s}\right]+\frac{\left(\mu-\delta_{2}(\log t)\right)}{\delta_{1} \delta_{2} \Gamma(\alpha-1)} \\
& \cdot \sum_{i=1}^{m-2} \beta_{i} \int_{1}^{\eta_{i}}\left(\log \frac{\eta_{i}}{s}\right)^{\alpha-2} h(s) \frac{d s}{s} .
\end{aligned}
$$

Lemma 8. The functions $G(t, s), \quad H\left(t, s ; \eta_{1}, \eta_{2}, \ldots, \eta_{m-2}\right)$ defined by (15) satisfy

(i) $G(t, s) \geq 0, H\left(t, s ; \eta_{1}, \eta_{2}, \ldots, \eta_{m-2}\right) \geq 0, \forall t, s \in[1, e]$,

(ii) $\min _{\tau_{1} \leq t \leq \tau_{2}} G(t, s) \geq \tau_{0} \max _{1 \leq t \leq e} G(t, s)=\tau_{0} G(s, s), \forall t$, $s \in(1, e), 1<\tau_{1}<\tau_{2}<e$, 


$$
\begin{aligned}
G(s, s) & =\frac{1}{\Gamma(\alpha)}\left(\log \frac{e}{s}\right)^{\alpha-1}, \\
\tau_{0} & =\min _{\tau_{1} \leq t \leq \tau_{2}} \psi(t)=\left(\log \frac{e}{\tau_{2}}\right),
\end{aligned}
$$

(iii) $N_{2} q(s) \leq H\left(t, s ; \eta_{1}, \eta_{2}, \ldots, \eta_{m-2}\right) \leq N_{1} q(s)$, where

$$
\begin{aligned}
q(s) & =\frac{(\log (e / s))^{\alpha-2}}{\delta_{1} \delta_{2} \Gamma(\alpha)}, \\
N_{1} & =\left[\delta_{1} \sum_{i=1}^{m-2} \gamma_{i}+(\alpha-1) \mu \sum_{i=1}^{m-2} \beta_{i}\right], \\
N_{2} & =\min \left\{\sum_{i=1}^{m-2} \gamma_{i}\left(\log \frac{e}{\eta_{i}}\right), \frac{\delta_{1} \sum_{i=1}^{m-2} \gamma_{i}}{(\alpha-1)}\right\},
\end{aligned}
$$

(iv) $\min _{\tau_{1} \leq t \leq \tau_{2}} H\left(t, s ; \eta_{1}, \eta_{2}, \ldots, \eta_{m-2}\right) \geq \tau^{*} \max _{1 \leq t \leq e} H(t$, $\left.s ; \eta_{1}, \eta_{2}, \ldots, \eta_{m-2}\right), s \in(1, e)$, where

$$
\tau^{*}=\frac{1}{\mu}\left[\mu-\delta_{2}\left(\log \tau_{2}\right)\right]<1, \quad 1<\tau_{1}<\tau_{2}<e .
$$

Proof. It is clear that (i) holds. So we prove that (ii) is true.

(ii) In view of the expression for $G(t, s)$, it follows that $G(t, s) \leq G(s, s)$ for all $s, t \in[1, e]$.
If $1 \leq s \leq t \leq e$, we have

$$
\begin{aligned}
& \frac{G(t, s)}{G(s, s)}=\frac{\left[(\log (e / s))^{\alpha-1}-(\log (t / s))^{\alpha-1}\right]}{(\log (e / s))^{\alpha-1}} \\
& =\frac{\left[(\log (e / s))(\log (e / s))^{\alpha-2}-(\log (t / s))(\log (t / s))^{\alpha-2}\right]}{(\log (e / s))^{\alpha-1}} \\
& \geq \frac{(\log (e / s))^{\alpha-2}[(\log (e / s))-(\log (t / s))]}{(\log (e / s))^{\alpha-1}}=\frac{(\log (e / t))}{(\log (e / s))} \\
& \geq\left(\log \frac{e}{t}\right):=\psi(t) .
\end{aligned}
$$

If $1 \leq t \leq s \leq e$, we have

$$
\frac{G(t, s)}{G(s, s)}=1 \geq \psi(t)
$$

Thus

$$
\begin{aligned}
& \max _{1 \leq t \leq e} G(t, s)=G(s, s), \\
& \psi(t) G(s, s) \leq G(t, s) \leq G(s, s),
\end{aligned}
$$

$$
\forall t, s \in(1, e) .
$$

Therefore,

$$
\begin{aligned}
& \min _{\tau_{1} \leq t \leq \tau_{2}} G(t, s) \geq \tau_{0} \max _{1 \leq t \leq e} G(t, s)=\tau_{0} G(s, s) \\
& \forall t, s \in(1, e), 1<\tau_{1}<\tau_{2}<e .
\end{aligned}
$$

(iii) If $1 \leq s \leq \eta_{i}, i=1,2, \ldots, m-2$,

$$
\begin{aligned}
H\left(t, s ; \eta_{1}, \eta_{2}, \ldots, \eta_{m-2}\right) & =\frac{\sum_{i=1}^{m-2} \gamma_{i}\left[(\log (e / s))^{\alpha-1}-\left(\log \left(\eta_{i} / s\right)\right)^{\alpha-1}\right]}{\delta_{2} \Gamma(\alpha)}+\frac{\left(\mu-\delta_{2}(\log t)\right) \sum_{i=1}^{m-2} \beta_{i}\left(\log \left(\eta_{i} / s\right)\right)^{\alpha-2}}{\delta_{1} \delta_{2} \Gamma(\alpha-1)} \\
& \leq \frac{\sum_{i=1}^{m-2} \gamma_{i}(\log (e / s))^{\alpha-1}}{\delta_{2} \Gamma(\alpha)}+\frac{\left(\mu-\delta_{2}(\log t)\right) \sum_{i=1}^{m-2} \beta_{i}\left(\log \left(\eta_{i} / s\right)\right)^{\alpha-2}}{\delta_{1} \delta_{2} \Gamma(\alpha-1)} \\
& =\frac{1}{\delta_{1} \delta_{2} \Gamma(\alpha)}\left[\delta_{1} \sum_{i=1}^{m-2} \gamma_{i}\left(\log \frac{e}{s}\right)^{\alpha-1}+(\alpha-1) \mu \sum_{i=1}^{m-2} \beta_{i}\left(\log \frac{\eta_{i}}{s}\right)^{\alpha-2}\right] \\
& \leq \frac{(\log (e / s))^{\alpha-2}}{\delta_{1} \delta_{2} \Gamma(\alpha)}\left[\delta_{1}\left(\log \frac{e}{s}\right)^{m-2} \sum_{i=1}^{m-2}+(\alpha-1) \mu \sum_{i=1}^{m-2} \beta_{i}\right] \\
& \leq \frac{(\log (e / s))^{\alpha-2}\left[\delta_{1} \sum_{i=1}^{m-2} \gamma_{i}+(\alpha-1) \mu \sum_{i=1}^{m-2} \beta_{i}\right]:=N_{1} q(s),}{\delta_{1} \delta_{2} \Gamma(\alpha)} \\
H\left(t, s ; \eta_{1}, \eta_{2}, \ldots, \eta_{m-2}\right) & =\frac{\sum_{i=1}^{m-2} \gamma_{i}\left[(\log (e / s))^{\alpha-1}-\left(\log \left(\eta_{i} / s\right)\right)^{\alpha-1}\right]}{\delta_{2} \Gamma(\alpha)}+\frac{\left(\mu-\delta_{2}(\log t)\right) \sum_{i=1}^{m-2} \beta_{i}\left(\log \left(\eta_{i} / s\right)\right)^{\alpha-2}}{\delta_{1} \delta_{2} \Gamma(\alpha-1)}
\end{aligned}
$$




$$
\begin{aligned}
& \geq \frac{\sum_{i=1}^{m-2} \gamma_{i}\left[(\log (e / s))(\log (e / s))^{\alpha-2}-\left(\log \left(\eta_{i} / s\right)\right)\left(\log \left(\eta_{i} / s\right)\right)^{\alpha-2}\right]}{\delta_{2} \Gamma(\alpha)} \\
& \geq \frac{(\log (e / s))^{\alpha-2}}{\delta_{2} \Gamma(\alpha)} \sum_{i=1}^{m-2} \gamma_{i}\left[\left(\log \frac{e}{s}\right)-\left(\log \frac{\eta_{i}}{s}\right)\right]=\frac{(\log (e / s))^{\alpha-2}}{\delta_{2} \Gamma(\alpha)} \sum_{i=1}^{m-2} \gamma_{i}\left(\log \frac{e}{\eta_{i}}\right):=N_{2} q(s) .
\end{aligned}
$$

$$
\begin{aligned}
& \text { If } \eta_{i} \leq s \leq e, i=1,2, \ldots, m-2 \text {, we have } \\
& \begin{aligned}
H\left(t, s ; \eta_{1}, \eta_{2}, \ldots, \eta_{m-2}\right)=\frac{\sum_{i=1}^{m-2} \gamma_{i}(\log (e / s))^{\alpha-1}}{\delta_{2} \Gamma(\alpha)} \\
\quad \leq \frac{(\log (e / s))^{\alpha-2}}{\delta_{1} \delta_{2} \Gamma(\alpha)} \delta_{1} \sum_{i=1}^{m-2} \gamma_{i} \leq \delta_{1} \sum_{i=1}^{m-2} \gamma_{i} q(s)<N_{1} q(s), \\
H\left(t, s ; \eta_{1}, \eta_{2}, \ldots, \eta_{m-2}\right)=\frac{\sum_{i=1}^{m-2} \gamma_{i}(\log (e / s))^{\alpha-1}}{\delta_{2} \Gamma(\alpha)} \\
\quad=\frac{\sum_{i=1}^{m-2} \gamma_{i}(\alpha-1)(\log (e / s))^{\alpha-1}}{\delta_{2}(\alpha-1) \Gamma(\alpha)} \\
\geq \frac{(\log (e / s))^{\alpha-2}}{\delta_{1} \delta_{2} \Gamma(\alpha)} \frac{\delta_{1} \sum_{i=1}^{m-2} \gamma_{i}}{(\alpha-1)} \geq N_{2} q(s) .
\end{aligned}
\end{aligned}
$$

(iv) Since $\partial H\left(t, s ; \eta_{1}, \eta_{2}, \ldots, \eta_{m-2}\right) / \partial t=-\sum_{i=1}^{m-2} \beta_{i}\left(\log \left(\eta_{i} / s\right)\right)^{\alpha-2} /$ $\delta_{1} \Gamma(\alpha-1) t \leq 0$, then $H\left(t, s ; \eta_{1}, \eta_{2}, \ldots, \eta_{m-2}\right)$ is nonincreasing in $t$, so

$$
\begin{aligned}
\min _{\tau_{1} \leq t \leq \tau_{2}} H\left(t, s ; \eta_{1}, \eta_{2}, \ldots, \eta_{m-2}\right) & \\
= & \frac{\sum_{i=1}^{m-2} \gamma_{i}\left[(\log (e / s))^{\alpha-1}-\left(\log \left(\eta_{i} / s\right)\right)^{\alpha-1}\right]}{\delta_{2} \Gamma(\alpha)} \\
& +\frac{\left(\mu-\delta_{2}\left(\log \tau_{2}\right)\right) \sum_{i=1}^{m-2} \beta_{i}\left(\log \left(\eta_{i} / s\right)\right)^{\alpha-2}}{\delta_{1} \delta_{2} \Gamma(\alpha-1)} \\
= & \frac{\sum_{i=1}^{m-2} \gamma_{i}\left[(\log (e / s))^{\alpha-1}-\left(\log \left(\eta_{i} / s\right)\right)^{\alpha-1}\right]}{\delta_{2} \Gamma(\alpha)} \\
& +\tau^{*} \frac{\mu \sum_{i=1}^{m-2} \beta_{i}\left(\log \left(\eta_{i} / s\right)\right)^{\alpha-2}}{\delta_{1} \delta_{2} \Gamma(\alpha-1)} \\
\geq & \tau^{*} \max _{1 \leq t \leq e} H\left(t ; \eta_{1}, \eta_{2}, \ldots, \eta_{m-2}, s\right) .
\end{aligned}
$$

\section{Existence Results}

Let us denote by $E=C([1, e], R)$ the Banach space of all continuous functions from $[1, e]$ to $\mathbb{R}$ endowed with the norm $\|x\|=\operatorname{Sup}_{t \in[1, e]}|x(t)|$ and let $P$ be the cone $P=\{x \in E: x(t) \geq$ $0, t \in[1, e]\}$.

Through this paper, we assume that the function $f$ : $[1, e] \times[0, \infty) \rightarrow[0, \infty)$ satisfies the following conditions of Carathéodory type:
$\left(H_{1}\right)$ : (i) $f(t, x)$ is Lebesgue measurable with respect to $t$ on $[1, e]$,

(ii) $f(t, x)$ is continuous with respect to $x$ on $[0, \infty)$. By Lemma 6, we obtain an operator $\mathscr{F}: E \rightarrow E$ as

$$
\begin{array}{r}
(\mathscr{F} x)(t)=x(t) \\
=\int_{1}^{e}\left[G(t, s)+H\left(t, s ; \eta_{1}, \eta_{2}, \ldots, \eta_{m-2}\right)\right] h(s) \frac{d s}{s}, \\
t \in[1, e] .
\end{array}
$$

It should be noticed that problem (1) has solutions if and only if the operator $\mathscr{F}$ has fixed points.

The first existence and uniqueness result is based on the Banach contraction principle.

Theorem 9. Assume that the condition $\left(H_{1}\right)$ holds and there exists a real-valued function $g(t) \in L[1, e]$ such that

$$
\begin{aligned}
|f(t, x(t))-f(t, y(t))| \leq g(t)|x-y|, & \\
& \forall t \in[1, e], x, y \in \mathbb{R} .
\end{aligned}
$$

Then problem (1) has a unique solution provided $\|g\| \Psi<1$, where

$$
\Psi=\left[\frac{1}{\Gamma(\alpha+1)}+\frac{N_{1}}{\delta_{1} \delta_{2}(\alpha-1) \Gamma(\alpha)}\right]
$$

Proof. We set $\operatorname{Sup}_{t \in[1, e]}|f(s, 0)|=p<\infty$ and choose $\rho \geq$ $\Psi p /(1-\Psi\|g\|)$.

Now, we show that $\mathscr{F} \mathscr{B}_{\rho} \subset \mathscr{B}_{\rho}$, where $\mathscr{B}_{\rho}=\{x \in E$ : $\|x\| \leq \rho\}$. For any $x \in \mathscr{B}_{\rho}$, we have

$$
\begin{aligned}
& \|(\mathscr{F} x)(t)\| \\
& =\operatorname{Sup}_{t \in[1, e]}\left\{\int_{1}^{e}\left[G(t, s)+H\left(t, s ; \eta_{1}, \eta_{2}, \ldots, \eta_{m-2}\right)\right]\right. \\
& \left.\cdot f(s, x(s)) \frac{d s}{s}\right\} \leq \int_{1}^{e}\left(G(s, s)+N_{1} q(s)\right) \\
& \cdot(|f(s, x(s))-f(s, 0)|+|f(s, 0)|) \frac{d s}{s}
\end{aligned}
$$




$$
\begin{aligned}
& \leq \int_{1}^{e}\left(G(s, s)+N_{1} q(s)\right)(g(s) \rho+p) \frac{d s}{s} \\
& \leq(\|g\| \rho+p)\left[\frac{1}{\Gamma(\alpha)} \int_{1}^{e}\left(\log \frac{e}{s}\right)^{\alpha-1} \frac{d s}{s}\right. \\
& \left.+\frac{N_{1}}{\delta_{1} \delta_{2} \Gamma(\alpha)} \int_{1}^{e}\left(\log \frac{e}{s}\right)^{\alpha-2} \frac{d s}{s}\right] \leq(\|g\| \rho+p) \\
& \cdot\left[\frac{1}{\Gamma(\alpha+1)}+\frac{N_{1}}{\delta_{1} \delta_{2}(\alpha-1) \Gamma(\alpha)}\right] \leq \Psi(\|g\| \rho \\
& +p) \leq \rho .
\end{aligned}
$$

It follows that $\mathscr{F}_{\rho} \subset \mathscr{B}_{\rho}$. For $x, y \in E$ and for each $t \in$ $[1, e]$, we have

$$
\begin{aligned}
& |(\mathscr{F} x)(t)-(\mathscr{F} y)(t)| \\
& \quad=\mid \int_{1}^{e}\left[G(t, s)+H\left(t, s ; \eta_{1}, \eta_{2}, \ldots, \eta_{m-2}\right)\right] \\
& \cdot(f(s, x(s))-f(s, y(s))) \frac{d s}{s} \mid \leq \int_{1}^{e}(G(s, s) \\
& \left.\quad+N_{1} q(s)\right) g(s)|x(s)-y(s)| \frac{d s}{s} \leq\|g\|\|x-y\| \\
& \quad \cdot \int_{1}^{e}\left(G(s, s)+N_{1} q(s)\right) \frac{d s}{s} \leq \Psi\|g\|\|x-y\| .
\end{aligned}
$$

Hence it follows that $\|(\mathscr{F} x)(t)-(\mathscr{F} y)(t)\| \leq \Psi\|g\|\|x-y\|$, where $\Psi\|g\|<1$. Therefore $\mathscr{F}$ is a contraction. Hence by the contraction mapping principle, problem (1) has a uniqueness solution.

Theorem 10 (nonlinear alternative for single valued maps [13]). Let $E$ be a Banach space, $\mathscr{C}$ a closed, convex subset of E, $\mathcal{U}$ an open subset of $\mathscr{C}$, and $0 \in \mathcal{U}$. Suppose that $F: \overline{\mathcal{U}} \rightarrow \mathscr{C}$ is a continuous, compact (i.e., $F(\overline{\mathcal{U}})$ is a relatively compact subset of $\mathscr{C})$ map. Then either

(i) F has a fixed point in $\overline{\mathcal{U}}$ or

(ii) There is $x \in \partial \mathcal{U}$ (the boundary of $\mathscr{U}$ in $\mathscr{C}$ ) and $\lambda \epsilon$ $(1, e)$ with $x=\lambda F(x)$.

Theorem 11. Assume that $\left(H_{1}\right)$ and the following conditions hold:

$\left(\mathrm{H}_{2}\right)$ There exist two nonnegative real-valued functions $h_{1}, h_{2} \in[1, e]$ such that

$$
\begin{aligned}
f(t, x) \leq h_{1}(t)+ & h_{2}(t) x, \\
& \text { for every } t \in[1, e] \text {, all } x \in[0, \infty) .
\end{aligned}
$$

$\left(H_{3}\right)$ There exists a constant $M>0$, such that

$$
\frac{M}{\left(\left\|h_{1}\right\|+\left\|h_{2}\right\| M\right) \Psi}>1 .
$$

Then the boundary value problem (1) has at least one solution on $[1, e]$.
Proof. First, we show that the operator $\mathscr{F}: P \rightarrow P$ is continuous.

For any $x_{n}, x \in P, n=1,2, \ldots$ with $\lim _{n \rightarrow \infty} x_{n}(t)=$ $x(t), t \in[1, e]$. Thus, by condition (ii) of $\left(H_{1}\right)$, we have $\lim _{n \rightarrow \infty} f\left(t, x_{n}(t)\right)=f(t, x(t)), t \in[1, e]$. So, we can conclude that

$$
\operatorname{Sup}_{t \in[1, e]}\left|f\left(t, x_{n}(t)\right)-f(t, x(t))\right| \longrightarrow 0, \quad \text { as } n \longrightarrow \infty \text {. }
$$

On the other hand,

$$
\begin{aligned}
& \left|\left(\mathscr{F} x_{n}\right)(t)-(\mathscr{F} x)(t)\right| \\
& \quad=\mid \int_{1}^{e}\left[G(t, s)+H\left(t, s ; \eta_{1}, \eta_{2}, \ldots, \eta_{m-2}\right)\right] \\
& \quad \cdot(f(s, x(s))-f(s, y(s))) \frac{d s}{s} \mid \\
& \quad \leq \operatorname{Sup}_{t \in[1, e]}\left|f\left(t, x_{n}(t)\right)-f(t, x(t))\right| \int_{1}^{e}(G(s, s) \\
& \left.\quad+N_{1} q(s)\right) \frac{d s}{s} \leq\left[\frac{1}{\Gamma(\alpha+1)}\right. \\
& \left.\quad+\frac{N_{1}}{\delta_{1} \delta_{2}(\alpha-1) \Gamma(\alpha)}\right]_{t \in[1, e]} \mid f\left(t, x_{n}(t)\right) \\
& \quad-f(t, x(t)) \mid .
\end{aligned}
$$

Hence,

$$
\left\|\left(\mathscr{F} x_{n}\right)(t)-(\mathscr{F} x)(t)\right\| \longrightarrow 0 \text { as } n \longrightarrow \infty
$$

This means that $\mathscr{F}$ is continuous.

Now, we show that $\mathscr{F}$ maps bounded sets into bounded sets in $P$. It suffices to show that, for any $\sigma>0$, there exists a positive constant $\rho_{1}>0$ such that, for each $x \in \mathscr{B}_{\rho^{*}}=\{x \in$ $\left.P:\|x\| \leq \rho^{*}\right\}$, we have $\|\mathscr{F} x\| \leq \rho_{1}$. By (33), for each $t \in[1, e]$, we have

$$
\begin{aligned}
& |(\mathscr{F} x)(t)|=\mid \int_{1}^{e}\left[G(t, s)+H\left(t, s ; \eta_{1}, \eta_{2}, \ldots, \eta_{m-2}\right)\right] \\
& \cdot f(s, x(s)) \frac{d s}{s} \mid \leq \int_{1}^{e}\left[G(s, s)+N_{1} q(s)\right]\left(h_{1}(s)\right. \\
& \left.+h_{2}(s) x(s)\right) \frac{d s}{s} \leq\left(\left\|h_{1}\right\|+\left\|h_{2}\right\| \rho^{*}\right) \\
& \cdot \int_{1}^{e}\left(G(s, s)+N_{1} q(s)\right) \frac{d s}{s} \leq\left(\left\|h_{1}\right\|+\left\|h_{2}\right\| \rho^{*}\right) \\
& \cdot\left[\frac{1}{\Gamma(\alpha+1)}+\frac{N_{1}}{\delta_{1} \delta_{2}(\alpha-1) \Gamma(\alpha)}\right] \leq\left(\left\|h_{1}\right\|+\left\|h_{2}\right\|\right. \\
& \left.\cdot \rho^{*}\right) \Psi:=\rho_{1},
\end{aligned}
$$


which implies that $\|(\mathscr{F} x)\| \leq \rho_{1}$. Further, we let $t_{1}, t_{2} \in[1, e]$ with $t_{1}<t_{2}$ and $x \in \mathscr{B}_{\rho^{*}}$, where $\mathscr{B}_{\rho^{*}}$ is a bounded set of $P$, and then we find that

$$
\begin{aligned}
& \left|\left({ }_{H}^{C} D \mathscr{F} x\right)(t)\right|=\mid-\frac{1}{\Gamma(\alpha-1)} \\
& \cdot \int_{1}^{e}\left[\left(\log \frac{t}{s}\right)^{\alpha-2}+\frac{1}{\delta_{1}} \sum_{i=1}^{m-2} \beta_{i}\left(\log \frac{\eta_{i}}{s}\right)^{\alpha-2}\right] \\
& \cdot f(s, x(s)) \frac{d s}{s} \mid \leq\left(\left\|h_{1}\right\|+\left\|h_{2}\right\| \rho^{*}\right) \\
& \cdot \frac{\left(\delta_{1}+\sum_{i=1}^{m-2} \beta_{i}\right)}{\delta_{1} \Gamma(\alpha-1)}:=\rho_{2} .
\end{aligned}
$$

Hence, for $t_{1}, t_{2} \in[1, e]$, we have

$$
\begin{aligned}
& \left|(\mathscr{F} x)\left(t_{2}\right)-(\mathscr{F} x)\left(t_{1}\right)\right| \leq \int_{t_{1}}^{t_{2}}\left({ }_{H}^{C} D \mathscr{F} x\right)(s) d s \\
& \quad \leq \rho_{2}\left(t_{2}-t_{1}\right) .
\end{aligned}
$$

This implies that $\mathscr{F}$ maps bounded sets into equicontinuous sets of $P$.

Thus, by the Arzelà-Ascoli theorem, the operator $\mathscr{F}$ : $P \rightarrow P$ is completely continuous.

Next, we consider the set $V=\{u \in E \mid x=\mu \mathscr{F} x, 0<$ $\mu<1\}$ and show that the set $V$ is bounded, let $x \in V$, and then $x=\mu \mathscr{F} x, 0<\mu<1$. For any $t \in[1, e]$, we have

$$
\begin{aligned}
& |x(t)|=\mu|(\mathscr{F} x)(t)| \\
& \leq \int_{1}^{e}\left[G(t, s)+H\left(t, s ; \eta_{1}, \eta_{2}, \ldots, \eta_{m-2}\right)\right] \\
& \quad \cdot|f(s, x(s))| \frac{d s}{s} \leq \rho_{1} .
\end{aligned}
$$

Thus, $\|x(t)\| \leq \rho_{1}$ for any $t \in[1, e]$, so that set $V$ is bounded. Thus, by the conclusion of Theorem 10, the operator $\mathscr{F}$ has at least one fixed point, which implies that problem (1) has at least one solution.

Theorem 12 (Krasnoselskii fixed point theorem [14]). Let $E$ be a Banach space and $K \subset E$ is a cone in E. Assume that $\Omega_{1}$ and $\Omega_{2}$ are open subsets of $E$ with $0 \in \Omega_{1}$ and $\bar{\Omega}_{1} \subset \Omega_{2}$. Let $T: K \cap\left(\bar{\Omega}_{2} \backslash \Omega_{1}\right) \rightarrow K$ be completely continuous operator. In addition suppose that either

(i) $\|T u\| \leq\|u\|, \forall u \in K \cap \partial \Omega_{1}$ and $\|T u\| \geq\|u\|, \forall u \in$ $K \cap \partial \Omega_{2}$ or

(ii) $\|T u\| \leq\|u\|, \forall u \in K \cap \partial \Omega_{2}$ and $\|T u\| \geq\|u\|, \forall u \in$ $K \cap \partial \Omega_{1}$

holds. Then $T$ has a fixed point in $K \cap\left(\bar{\Omega}_{2} \backslash \Omega_{1}\right)$.
To state the last result of this section, we set

$$
\begin{aligned}
M_{1} & =(\Psi)^{-1}, \\
M_{2} & =\left(\frac{\tau_{0}}{\Gamma(\alpha+1)}\right. \\
& \left.+(\alpha-1) \tau^{*}\left[\frac{\mu \sum_{i=1}^{m-2} \beta_{i}}{\delta_{1} \delta_{2} \Gamma(\alpha)}-\frac{\sum_{i=1}^{m-2} \gamma_{i}}{\delta_{2} \Gamma(\alpha+1)}\right]\right)^{-1} .
\end{aligned}
$$

Theorem 13. Suppose that there exist two positive constants $r_{2}>r_{1}>0$ and $M_{1} \in\left(0, M_{2}\right)$ :

(i) $f(t, x) \leq M_{1} r_{2}$, for $(t, x) \in[1, e] \times\left[0, r_{2}\right]$.

(ii) $f(t, x) \geq M_{2} r_{1}$, for $(t, x) \in[1, e] \times\left[0, r_{1}\right]$.

Then (1) has at least a positive solution.

Proof. Let $\Omega_{i}=\left\{x \in E \mid\|x\|<r_{i}\right\}, i=1,2$. From the proof of Theorem 11, we know that the operator $\mathscr{F}$ defined by (28) is completely continuous on $P$.

For any $x \in P \cap \partial \Omega_{1}$, it follows that

$$
\begin{aligned}
& \|(\mathscr{F} x)(t)\| \\
& =\operatorname{Sup}_{t \in[1, e]}\left\{\int_{1}^{e}\left[G(t, s)+H\left(t, s ; \eta_{1}, \eta_{2}, \ldots, \eta_{m-2}\right)\right]\right. \\
& \left.\cdot f(s, x(s)) \frac{d s}{s}\right\} \geq \int_{1}^{e}\left(\tau_{0} G(s, s)\right. \\
& \left.+\tau^{*} H\left(1, s ; \eta_{1}, \eta_{2}, \ldots, \eta_{m-2}\right)\right) M_{2} r_{1} \frac{d s}{s} \\
& \geq M_{2} r_{1}\left[\frac{\tau_{0}}{\Gamma(\alpha+1)}+(\alpha-1) \tau^{*}\left[\frac{\mu \sum_{i=1}^{m-2} \beta_{i}}{\delta_{1} \delta_{2} \Gamma(\alpha)}\right.\right. \\
& \left.\left.-\frac{\sum_{i=1}^{m-2} \gamma_{i}}{\delta_{2} \Gamma(\alpha+1)}\right]\right] \geq r_{1}=\|x\| ;
\end{aligned}
$$

that is, $\|(\mathscr{F} x)(t)\| \geq\|x\|, \quad x \in P \cap \partial \Omega_{1}$.

On the other hand, for any $x \in P \cap \partial \Omega_{2}$, it follows that

$$
\begin{aligned}
& \|(\mathscr{F} x)(t)\| \\
& =\operatorname{Sup}_{t \in[1, e]}\left\{\int_{1}^{e}\left[G(t, s)+H\left(t, s ; \eta_{1}, \eta_{2}, \ldots, \eta_{m-2}\right)\right]\right. \\
& \left.\cdot f(s, x(s)) \frac{d s}{s}\right\} \leq \int_{1}^{e}\left(G(s, s)+N_{1} q(s)\right) \\
& \cdot M_{1} r_{2} \frac{d s}{s} \leq M_{1} r_{2} \Psi=r_{2}=\|x\| ;
\end{aligned}
$$

that is, $\|(\mathscr{F} x)(t)\| \leq\|x\|, \quad x \in P \cap \partial \Omega_{2}$.

In view of Theorem $12, \mathscr{F}$ has a fixed point in $P \cap\left(\bar{\Omega}_{2} / \Omega_{1}\right)$, which is a positive solution to (1).

\section{Examples}

In this section, we exemplify our theoretical results obtained in Section 3. 
Example 1. Consider the following BVP for Hadamard fractional differential equation:

$$
\begin{aligned}
{ }_{H}^{C} D^{7 / 3} x(t)+f(t, x(t)) & =0, \quad 1 \leq t \leq e, \\
{ }_{H}^{C} D x(1) & =\frac{1}{2}{ }_{H}^{C} D x\left(\frac{4}{3}\right), \\
{ }_{H}^{C} D^{2} x(1) & =0, \\
x(e) & =\frac{1}{4} x\left(\frac{4}{3}\right) .
\end{aligned}
$$

Here $\alpha=7 / 3, \beta=0.5, \gamma=0.25, \eta=4 / 3$, and

$$
\begin{aligned}
f(t, x)=\frac{e^{t}}{2\left(e^{t}+1\right)}\left(\frac{x^{2}}{x+1}+\right. & \left.\frac{x}{4(x+1)}+\frac{3}{4}\right), \\
& (t, x) \in[1, e] \times[0, \infty) .
\end{aligned}
$$

Using the given data, we find that $\Psi=1.609, \delta_{1}=0.5, \delta_{2}=$ $0.75, \mu=0.928$, and

$$
\begin{array}{r}
|f(t, x)-f(t, y)| \leq \frac{e^{t}}{2\left(e^{t}+1\right)}|x-y| \leq \frac{1}{2}|x-y| \\
\text { with } g(t)=\frac{e^{t}}{2\left(e^{t}+1\right)}
\end{array}
$$

Hence, we obtain $\|g\| \Psi=0.805<1$. Therefore, by Theorem 9, problem (45) has a unique solution on $[1, e]$.

Example 2. Consider the following BVP for Hadamard fractional differential equation:

$$
\begin{aligned}
{ }_{H}^{C} D^{5 / 2} x(t)+f(t, x(t)) & =0, \quad 1 \leq t \leq e, \\
{ }_{H}^{C} D x(1) & =\frac{1}{4}{ }_{H}^{C} D x\left(\frac{3}{2}\right), \\
{ }_{H}^{C} D^{2} x(1) & =0, \\
x(e) & =\frac{1}{3} x\left(\frac{3}{2}\right) .
\end{aligned}
$$

Here $\alpha=5 / 2, \beta=0.25, \gamma=1 / 3, \eta=3 / 2$. Let

$$
\begin{aligned}
& f(t, x)=9 \sin ^{2} t+\frac{e^{t} x}{12\left(e^{t}+1\right)\left(x^{2}+1\right)}, \\
&(t, x) \in[1, e] \times[0, \infty),
\end{aligned}
$$

with $h_{1}(t)=9 \sin ^{2} t, h_{2}(t)=e^{t} / 12\left(e^{t}+1\right)$. Here, $f(t, x) \leq$ $h_{1}(t)+h_{2}(t) x$.

It is easy to verify that $M /\left(\left\|h_{1}\right\|+\left\|h_{2}\right\| M\right) \Psi=1.147>1$.

Then, by Theorem 11, problem (48) has at least one solution on $[1, e]$.
Example 3. Consider the following BVP for Hadamard fractional differential equation:

$$
\begin{aligned}
& { }_{H}^{C} D^{7 / 2} x(t)+f(t, x(t))=0, \quad 1 \leq t \leq e, \\
& { }_{H}^{C} D x(1)=\frac{1}{2}{ }_{H}^{C} D x\left(\frac{4}{3}\right)+\frac{1}{4}{ }_{H}^{C} D x\left(\frac{5}{2}\right), \\
& { }_{H}^{C} D^{2} x(1)=0, \\
& x(e)=\frac{1}{3} x\left(\frac{4}{3}\right)+\frac{1}{5} x\left(\frac{5}{2}\right),
\end{aligned}
$$

where $\alpha=7 / 2, \beta_{1}=0.5, \beta_{2}=0.25, \gamma_{1}=1 / 3, \gamma_{2}=1 / 5$, $\eta_{1}=4 / 3, \eta_{2}=5 / 2$. Let

$$
f(t, x)=\frac{1}{4} x+\frac{e^{t}}{5\left(e^{t}+1\right)}, \quad(t, x) \in[1, e] \times[0, \infty) .
$$

It is easy to verify that

$$
\begin{aligned}
M_{1} & =(\Psi)^{-1}=0.6196 \\
M_{2} & =\left(\frac{\tau_{0}}{\Gamma(\alpha+1)}\right. \\
& \left.+(\alpha-1) \tau^{*}\left[\frac{\mu \sum_{i=1}^{m-2} \beta_{i}}{\delta_{1} \delta_{2} \Gamma(\alpha)}-\frac{\sum_{i=1}^{m-2} \gamma_{i}}{\delta_{2} \Gamma(\alpha+1)}\right]\right)^{-1} \\
& =0.521 .
\end{aligned}
$$

Choosing $r_{1}=1 / 5, r_{2}=e$, we have

$$
\begin{array}{ll}
f(t \cdot x) \leq 1.2 \leq M_{1} r_{2}, & (t, x) \in[1, e] \times\left[0, r_{2}\right], \\
f(t \cdot x)>0.5 \geq M_{2} r_{1}, & (t, x) \in[1, e] \times\left[0, r_{1}\right] .
\end{array}
$$

Hence all conditions of Theorem 13 are satisfied; then problem (50) has at least one positive solution $x$ such that $1 / 5<$ $\|x\|<e$.

\section{Competing Interests}

The author declares no competing interests.

\section{References}

[1] Y. Y. Gambo, F. Jarad, D. Baleanu, and T. Abdeljawad, "On Caputo modification of the Hadamard fractional derivatives," Advances in Difference Equations, vol. 2014, article 10, 12 pages, 2014.

[2] B. Ahmad, S. K. Ntouyas, and A. Alsaedi, "New results for boundary value problems of Hadamard-type fractional differential inclusions and integral boundary conditions," Boundary Value Problems, vol. 275, p. 14, 2013.

[3] J. Tariboon, S. K. Ntouyas, and W. Sudsutad, "Nonlocal Hadamard fractional integral conditions for nonlinear Riemann-Liouville fractional differential equations," Boundary Value Problems, vol. 2014, article 253, 2014. 
[4] P. Thiramanus, S. K. Ntouyas, and J. Tariboon, "Existence and uniqueness results for Hadamard-type fractional differential equations with nonlocal fractional integral boundary conditions," Abstract and Applied Analysis, vol. 2014, Article ID 902054, 9 pages, 2014.

[5] B. Ahmad and S. K. Ntouyas, "An existence theorem for fractional hybrid differential inclusions of Hadamard type with Dirichlet boundary conditions," Abstract and Applied Analysis, vol. 2014, Article ID 705809, 7 pages, 2014.

[6] B. Ahmad and S. K. Ntouyas, "A fully Hadamard type integral boundary value problem of a coupled system of fractional differential equations," Fractional Calculus and Applied Analysis. An International Journal for Theory and Applications, vol. 17, no. 2, pp. 348-360, 2014.

[7] F. Jarad, T. Abdeljawad, and D. Baleanu, "Caputo-type modification of the Hadamard fractional derivatives," Advances in Difference Equations, vol. 2012, no. 1, article 142, 8 pages, 2012.

[8] A. A. Kilbas, "Hadamard-type fractional calculus," Journal of the Korean Mathematical Society, vol. 38, no. 6, pp. 1191-1204, 2001.

[9] I. Podlubny, Fractional Differential Equations, Academic Press, San Diego, Calif, USA, 1999.

[10] S. Pooseh, R. Almeida, and D. F. M. Torres, "Expansion formulas in terms of integer-order derivatives for the Hadamard fractional integral and derivative," Numerical Functional Analysis and Optimization, vol. 33, no. 3, pp. 301-319, 2012.

[11] A. A. Kilbas, H. M. Srivastava, and J. J. Trujillo, Theory and Applications of Fractional Differential Equations, vol. 204, Elsevier Science, Amsterdam, The Netherlands, 2006.

[12] S. G. Samko, A. A. Kilbas, and O. I. Marichev, Fractional Integrals and Derivatives, Gordon and Breach Science, Yverdon, Switzerland, 1993.

[13] B. Ahmad, S. K. Ntouyas, and J. Tariboon, "Existence results for mixed Hadamard and Riemann-Liouville fractional integrodifferential equations," Advances in Difference Equations, vol. 2015, no. 1, article 293, 8 pages, 2015.

[14] R. P. Agarwal, M. Meehan, and D. O'Regan, Fixed Point Theory and Applications, vol. 141, Cambridge University Press, Cambridge, UK, 2001. 


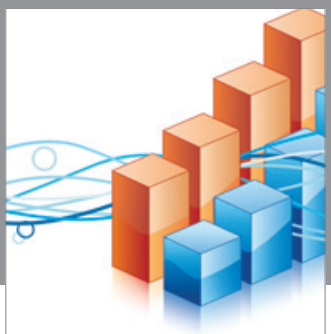

Advances in

Operations Research

vatem alat4

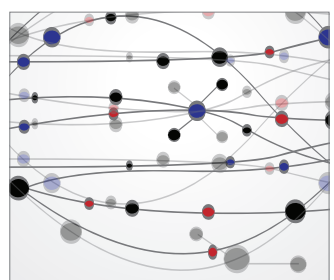

\section{The Scientific} World Journal
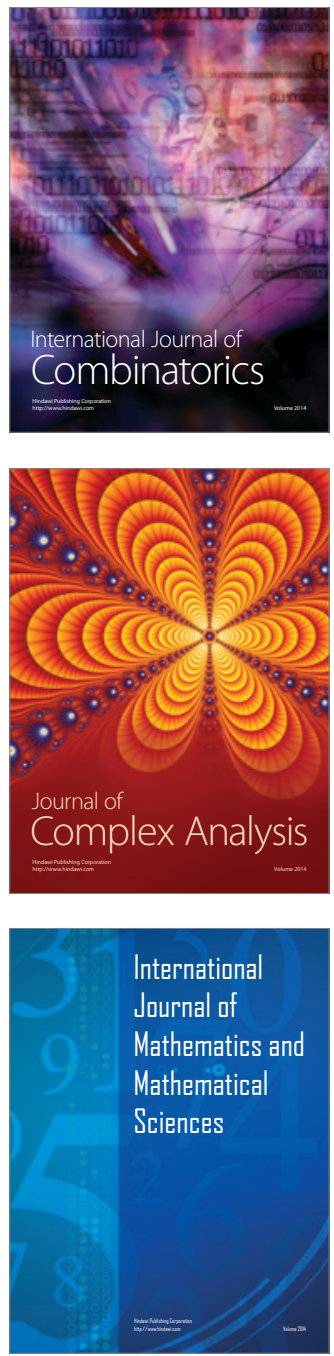
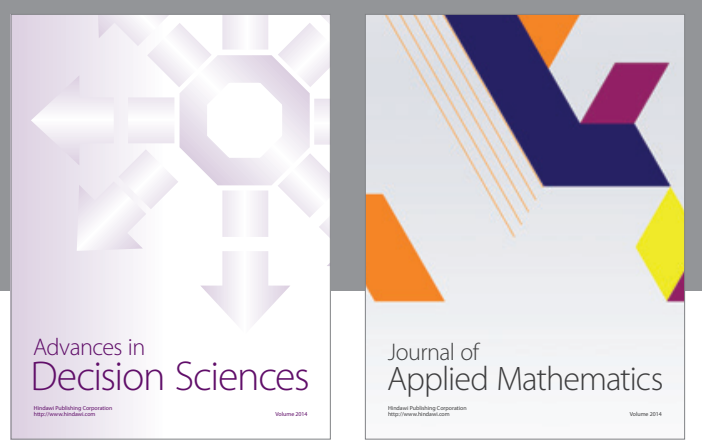

Algebra

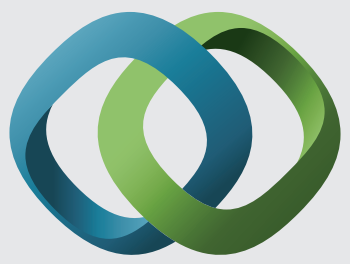

\section{Hindawi}

Submit your manuscripts at

http://www.hindawi.com
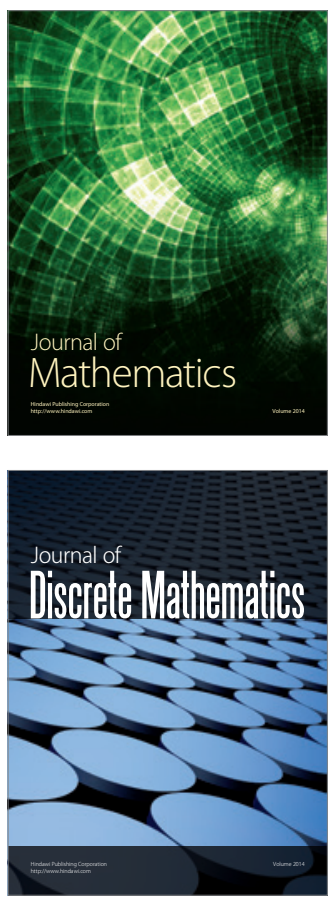

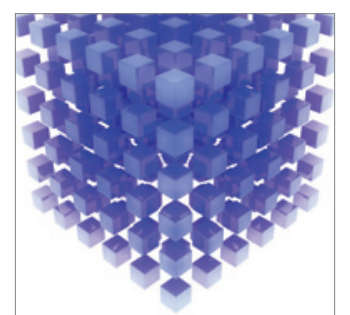

Mathematical Problems in Engineering
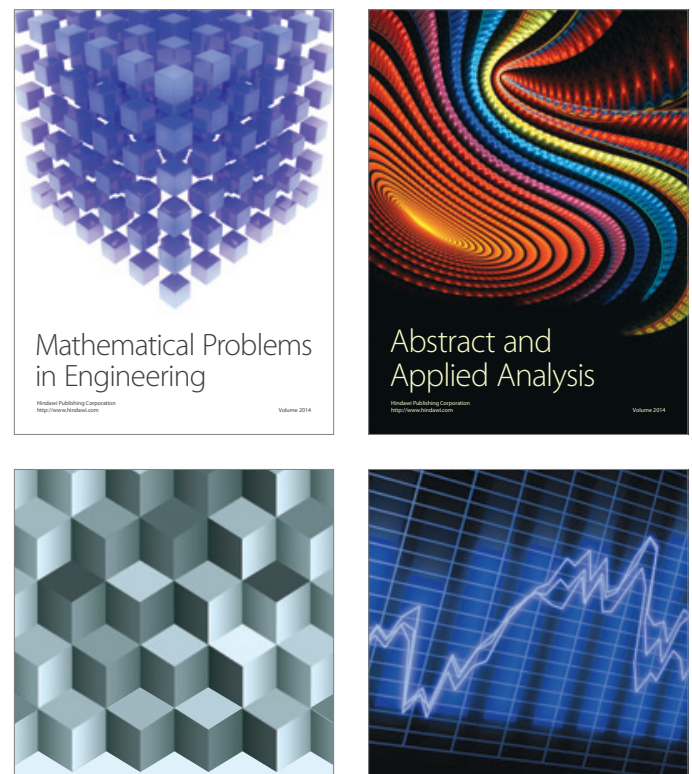

Journal of

Function Spaces

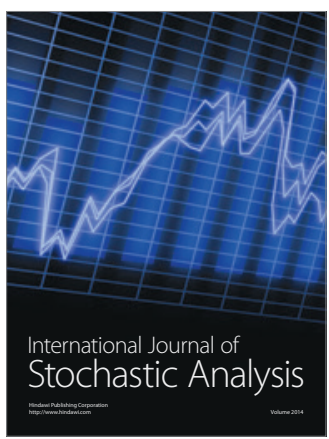

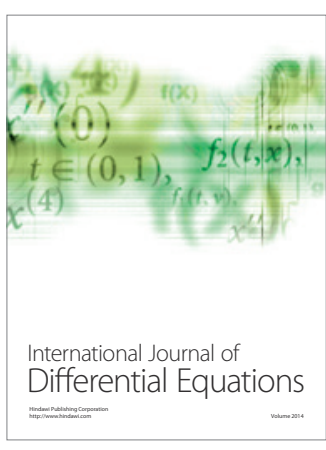
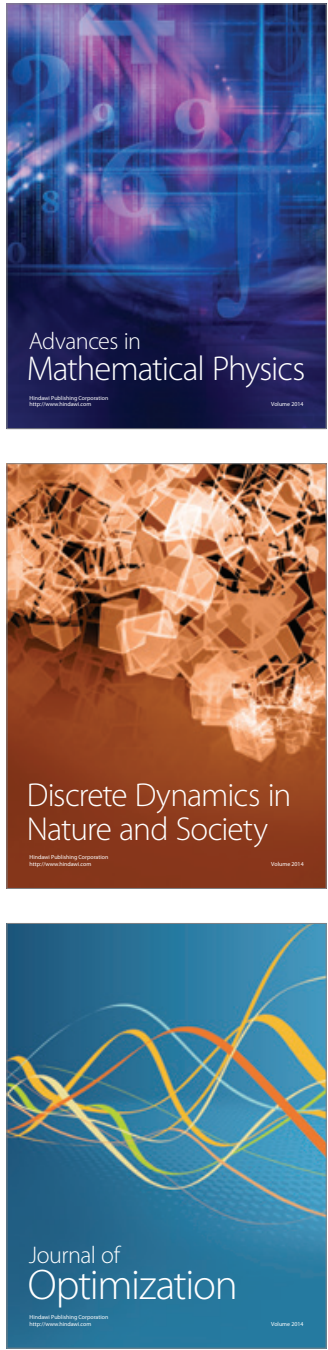